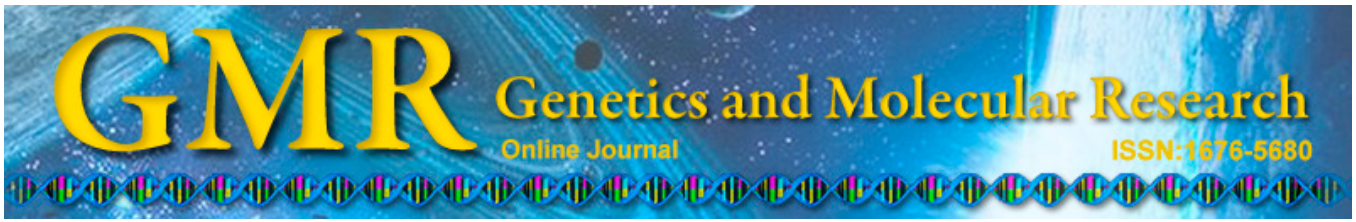

\title{
H3K27me3 may be associated with Oct4 and Sox 2 in mouse preimplantation embryos
}

F.-R. Wu, Y. Zhang, B. Ding, X.-H. Lei, J.-C. Huang, C.-H. Wang, Y. Liu, R. Wang and W.-Y. Li

School of Biological Science and Food Engineering,

Fuyang Teachers College,

Key Laboratory of Embryo Development and Reproductive Regulation, Anhui Province, Fuyang, China

Corresponding author: W.-Y. Li

E-mail: liwenyong@aliyun.com

Genet. Mol. Res. 13 (4): 10121-10129 (2014)

Received November 8, 2013

Accepted July 24, 2014

Published December 4, 2014

DOI http://dx.doi.org/10.4238/2014.December.4.6

\begin{abstract}
As a core member of polycomb repressive complex 2, the transcription and enzyme activity of enhancer of zeste homolog 2 (Ezh2) is directly involved in the trimethylation of lysine 27 on histone H3. In this study, the fluorescence intensity of H3K27me3 in mouse in vivo morulae and blastocysts was compared by indirect immunofluorescence staining. We found that demethylation of H3K27me3 occurred during the blastocyst stage. Real-time polymerase chain reaction was performed to investigate $E z h 2$ expression in oocytes and in preimplantation embryos. Ezh2 expression peaked during the zygote stage and gradually decreased from the 2-cell stage, exhibiting an inverse pattern when compared with Oct 4 and Sox 2 mRNA in mouse preimplantation embryos. To understand the role of developmentrelated genes on the transcription of mouse $E z h 2$, a promoter assay was performed in NIH/3T3 cells. Ezh2 expression was markedly suppressed by Oct 4 and Sox 2 alone in a dose-dependent manner, while Ezh2 promoter activity in co-transfection with Nanog, Klf-4, and c-Myc groups showed no significant change as compared with the control.
\end{abstract}


Our data suggest that the demethylation of $\mathrm{H} 3 \mathrm{~K} 27 \mathrm{me} 3$ is caused by the degressive expression and activity of Ezh2 in blastocysts, leading to increased expression of developmentally important transcription factors. We also observed negative effects of Oct 4 and Sox 2 on the transcription of Ezh2 and identified Oct4 and Sox 2 as novel negative regulators of Ezh2 at the post-translation level in a mouse preimplantation embryo.

Key words: Ezh2; Development-related genes; Mouse; Transcriptional regulation; Trimethylation of H3K27

\section{INTRODUCTION}

Trimethylation of lysine 27 on histone H3 (H3K27me3), which is catalyzed by polycomb repressive complex 2 (PRC2), is an important histone modification and considered to be an epigenetic marker of maternally derived chromatin mediating gene silencing in mammals (Park et al., 2009). Enhancer of zeste homolog 2 (Ezh2) is a histone methyltransferase and a core component of PRC2 (Sauvageau and Sauvageau, 2010). Several studies have indicated that the activity of Ezh2 may be associated with H3K27me3, but can also play key roles in embryonic development, tumor cell proliferation, and the physiology of several cell types and tissues. During mouse embryogenesis, Ezh2 is essential for establishing the first differentiated cells, the trophectoderm, and the pluripotent epiblast cells (Erhardt et al., 2003); its protein was also found to exhibit tissue specificity and depended on the embryo's stage of development (Zhu et al., 2011). In mouse somatic cloned embryos, inadequate expression of Ezh2 in inner cell mass (ICM) cells led to defective chromatin structure, particularly inadequate H3K27me3 (Zhang et al., 2009). In mouse and human, T-cell leukemia development was suppressed by Ezh2 and its associated genes (Simon et al., 2012). Moreover, Ezh2 is thought to be a therapeutic target for treating cancer (Qi et al., 2012).

Embryonic stem (ES) cells and induced pluripotent stem (iPS) cells are widely used in the study of developmental biology. The expression profiles of developmental genes, such as Oct4, Sox2, and Nanog, are closely related to the totipotency and pluripotency of ES and iPS cells, respectively (Boyer et al., 2005; Takahashi and Yamanaka, 2006). A recent study found that the epigenetic status of the Nanog promoter was directly regulated by Ezh2, indicating that Ezh2 can affect the equilibrium between self-renewal and differentiation in ES cells. In addition, the loss of Ezh2 and higher levels of Nanog are synchronously observed in iPS cells (Villasante et al., 2011). In murine Oct4(+)Sca-1(+)Lin(-)CD45(-) very small embryonic-like stem cells, similarly to other pluripotent stem cells, the pluripotent states are maintained through an Ezh2-dependent bivalent domain-mediated epigenetic mechanism (Shin et al., 2012). At the onset of transcription factor-induced reprogramming, iPS cells lacking Ezh2 efficiently silenced the somatic transcriptome and differentiated into tissues derived from the 3 germ layers (Fragola et al., 2013).

Although many studies have shown that Ezh2 and Ezh2-mediated H3K27me3 contribute to mammalian development and the status of ES and iPS cells, little is known regarding the upstream regulator of $E z h 2$ and the relationship with development-related genes. First, we examined H3K27me3 by using indirect immunofluorescence staining and by determining the expression pattern of Ezh2 using real-time polymerase chain reaction (PCR) in mouse preimplantation embryos and compared these results with the known expression patterns of 
Oct4, Sox2, and Nanog. Furthermore, we investigated whether the promoter activity of Ezh2 was influenced by these development-related genes to determine the pathway of early stage mammalian embryonic development.

\section{MATERIAL AND METHODS}

\section{Collection and culture of oocytes and embryos}

Kunming white mice were used as model in the present study. The purchasing and rearing methods and the collection and culture of oocytes and embryos were performed according to previously described methods (Wu et al., 2012a,b). MII stage oocytes, zygotes, 2-, 4-, 8-, and 16-cell embryos, morulae, and blastocysts were collected for this study as described previously (Wu et al., 2012a,b).

\section{Immunofluorescence histochemistry for detection of H3K27me3}

Morulae and blastocysts generated by in vivo fertilization were washed in $1 \mathrm{X}$ phosphate-buffered saline (PBS), fixed in 4\% paraformaldehyde in 1X PBS, and permeabilized in $0.2 \%$ Triton X-100 in PBS for $30 \mathrm{~min}$ at room temperature. After blocking in PBS containing $1 \%$ bovine serum albumin for $1 \mathrm{~h}$ at room temperature, the embryos were incubated overnight at $4^{\circ} \mathrm{C}$ in a 1:100 dilution of primary antibodies against H3K27me3 (Catalog No. ab6002, Abcam, Cambridge, UK). Subsequent steps were also performed as described previously ( $\mathrm{Wu}$ et al., 2012a). Samples without primary antibody were included as negative controls. Fluorescence was detected using a Leica confocal laser scanning microscope (TCS-SP5) according to manufacturer instructions.

\section{Real-time PCR}

Five zygotes or embryos were used for each time point and 5 samples (replicates) were collected from each stage. Total RNA was extracted and cDNAs were synthesized according to the protocols of the RNeasy Plus Micro Kit and Sensiscript RT Kit (Qiagen, Hilden, Germany). Quantitative PCR was carried out according to manufacturer instructions of FastStart Universal SYBR Green Master (Roche, Basel, Switzerland). The threshold cycle (Ct) was defined as the fractional cycle number by the method of global minimum. Gapdh was used as an internal control. The ratio change in the Ezh2 gene relative to Gapdh control gene was determined using the $2^{-\Delta \Delta \mathrm{Ct}}$ method. Data are reported as means $\pm \mathrm{SE}$ for the 3 replicates. The GraphPad Prism 5 software (GraphPad Software, San Diego, CA, USA) was used to identify significant differences. All primer sequences used for the present study are listed in Table 1.

\section{Cell culture, transfections, and luciferase assay}

Briefly, the promoter (-2106 bp) of the mouse Ezh2 gene was generated by PCR using the primers listed in Table 1 and subcloned into the pGL3-Basic Vector (Promega Co., Madison, WI, USA). Mouse Oct4, Sox2, Nanog, Klf-4, and c-Myc-pcDNA3.1 expression vectors (Invitrogen, Carlsbad, CA, USA) were used as described previously (Wu et al., 2012b). 
The constructs and orientation of the insert were confirmed by direct sequencing; plasmids used in transfection experiments were purified using a QIAfilter Plasmid Midi Kit (Qiagen). NIH/3T3 cells (mouse embryonic fibroblast cell line) were transfected using Lipofectamine (Invitrogen) with the following plasmids: 1) $500 \mathrm{ng}$ of the mouse Ezh2 promoter cloned into the pGL3-Basic luciferase reporter vector; 2) $100 \mathrm{ng} O c t 4$, sox2, Nanog, Klf-4, and c-Myc -pcDNA3.1 expression plasmid (Invitrogen); and 3) pRL-TK (Promega), at $100 \mathrm{ng} / \mathrm{well}$. Cell culture, transient transfections, luciferase assays, and statistical analysis were performed as reported previously (Wu et al., 2012b).

\section{Table 1. List of primer sequences used in real-time PCR and promoter analyses.}

\begin{tabular}{lll}
\hline Primer & Sequence $\left(3^{\prime}-5^{\prime}\right)$ & Purpose \\
\hline Ezh2-F & ACCAAGAGTGGAAGCAGCGG & For real-time PCR \\
Ezh2-R & CACTGGTGACTGAACACTCCCT & \\
Gapdh-F & ATTCAACGGCACAGTCAAGG & \\
Gapdh-R & GGTCCTCAGTGTAGCCCAAGA & For promoter assay \\
Ezh2-PF & CGACGCGTTCGCATCCTTTCATCTATTTTAC & For pcDNA3.1 expression plasmid \\
Ezh2-PR & CCCAAGCTTGCAGATAAAGTTAGTGCGGTTC & \\
Oct4-F & CGGAATTCGCCACCATGGCTGGACACCTGG & \\
Oct4-R & CCGCTCGAGTCAGTTTGAATGCATG & \\
c-Myc-F & CGGGATCCGCCACCATGAGTGTGGGTCTT & \\
c-Myc-R & CCGCTCGAGTCATATTCACCTGG & \\
Nanog-F & CGGAATTCGCCACCATGCCCCTCAACGTG & \\
Nanog-R & CCGCTCGAGTTATGCACCAGAGTT & \\
Sox2-F & CGGGATCCGCCACCATGTATAACATGATGGA & \\
Sox2-R & CCGCTCGAGTCACATGTGCGACAGGGGC & \\
Klf-4-F & CGGGATCCGCCACCATGAGGCAGCCACCTGGC & \\
Klf-4-R & CCGCTCGAGTTAAAAGTGCCTCTTCATGT & \\
\hline
\end{tabular}

Underlined bases show the restriction sites.

\section{RESULTS}

\section{Modification of H3K27me3 in morulaes and blastocysts of in vivo mouse embryos}

Several in vivo morulae and blastocysts were collected to confirm the modification of H3K27me3. As shown in Figure 1, a dramatic change in H3K27me3 was observed between morulae and blastocysts. H3K27me3 was positively detected in morulaes, while very weak signals were observed in blastocysts. H3K27me3 modifications gradually disappeared from the morula stage; demethylation of $\mathrm{H} 3 \mathrm{~K} 27 \mathrm{me} 3$ occurred at this stage.

\section{Expression patterns of Ezh2 in mouse preimplantation embryos}

The expression patterns of $E z h 2$ in mouse MII oocytes and preimplantation embryos were detected by real-time PCR. Ezh2 was expressed more strongly in zygotes than in oocytes. As shown in Figure 2, the levels of Ezh2 gradually decreased from the 2-cell stage. At the blastocyst stage, weaker but persistent expression of Ezh2 was also detected. As a core member of PRC2 that catalyzes H3K27me3, the activity of mouse Ezh2 must be the lowest in blastocysts. These results are also consistent with the modification of H3K27me3 as shown in Figure 1. 


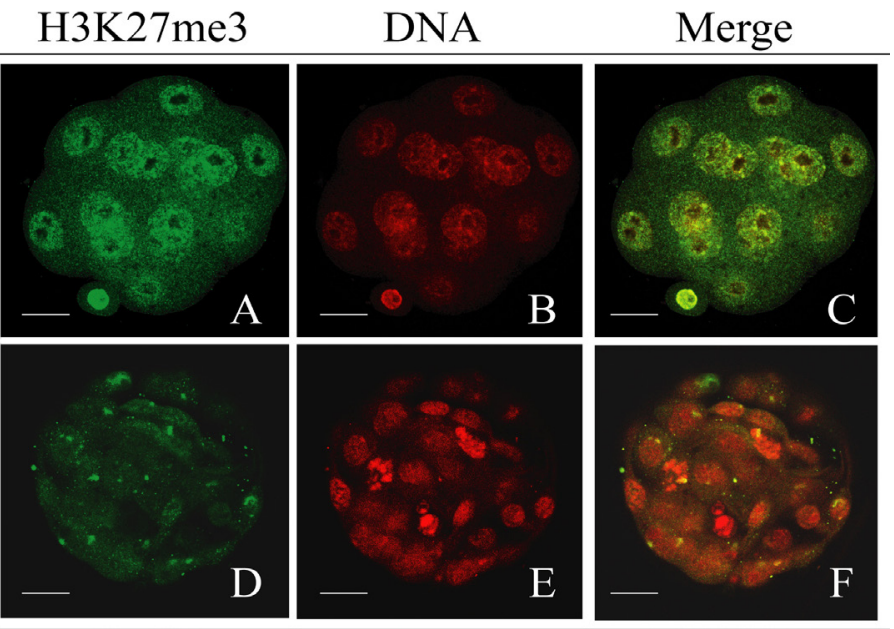

Figure 1. Trimethylation of lysine 27 on histone $3(\mathrm{H} 3 \mathrm{~K} 27 \mathrm{me} 3)$ in morulae and blastocysts of in vivo mouse embryos. Embryos were immunostained with specific antibodies against H3K27me3. H3K27me3, green; DNA, red. Scale bar $=20 \mu \mathrm{m}$.

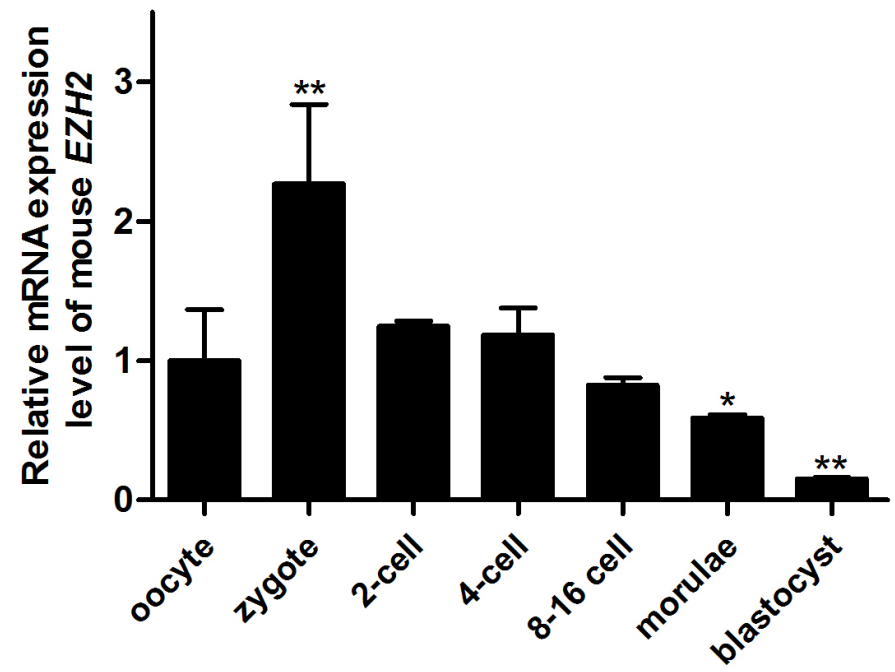

Figure 2. Expression patterns of mouse Ezh2 in MII stage oocytes and preimplantation embryos by real-time PCR. Data are reported as means $\pm \mathrm{SE}$ for 3 replicates. $* \mathrm{P}<0.05 ; * * \mathrm{P}<0.01$ as compared with oocytes.

\section{Promoter analysis of mouse Ezh2}

A 2106-bp 5'-flanking of mouse Ezh2 was cloned into the pGL-3 basic vector, and promoter analysis was performed to investigate the effect of Oct4, Sox2, Nanog, Klf-4, and c-Myc on the promoter activity of mouse Ezh2. The results showed that activity of the mouse Ezh2 promoter was downregulated by Oct 4 and Sox 2 alone in NIH/3T3 cells, while Ezh2 promoter activity in cells co-transfected with Nanog, Klf-4, and c-Myc groups did not show any significant change compared with the control (Mock) (Figure 3). To further examine whether 
the transcription of Ezh2 was regulated by Oct 4 and Sox 2 in a dose-dependent manner, 10-200 ng Oct4 and Sox 2 expression vectors were co-transfected with the mouse Ezh2 promoter. As shown in Figure 4, the inhibitory effects on the mouse Ezh2 promoter were gradually tightened when the amounts of Oct 4 and Sox 2 were increased; this indicates that mouse Ezh2 expression was repressed by Oct4 and Sox 2 in a dose-dependent manner (Figure 4).

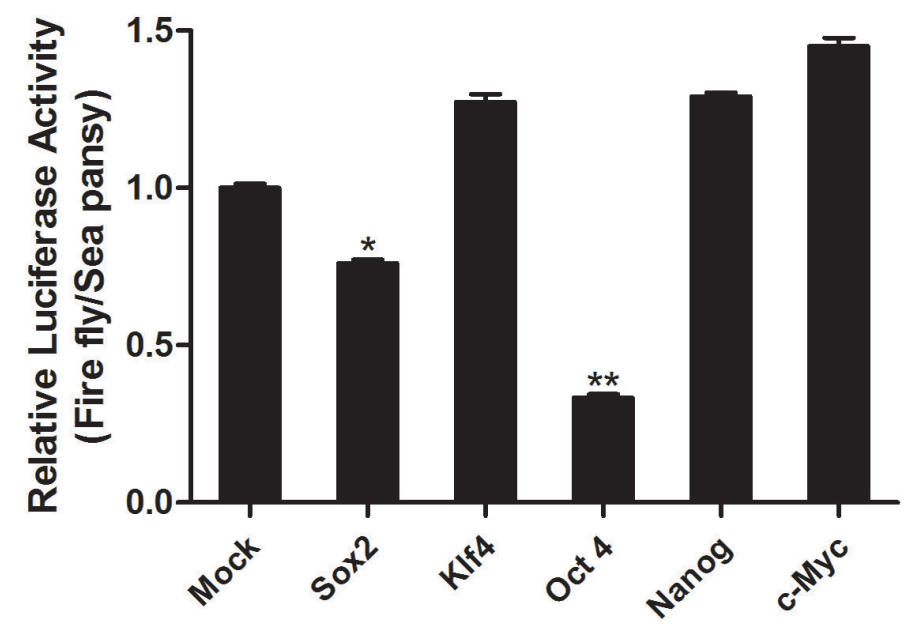

Figure 3. Promoter analysis of mouse $E z h 2$ in NIH/3T3 cells by luciferase assay. Oct4, c-Myc, Nanog, Klf-4, and $c$-Myc-pcDNA3.1 (100 ng) were co-transfected with mouse Ezh2 promoter (500 ng/well) into NTH/3T3 cells. The total amount of transfected plasmid, including the pRL-TK control vector (100 ng/well), was adjusted to 1.0 $\mu \mathrm{g}$ with empty vectors. Firefly and Renilla luciferase activities were measured $48 \mathrm{~h}$ after transfection. Relative luciferase activity was calculated by dividing firefly luciferase activity with Renilla luciferase activity. Results are reported as means $\pm \mathrm{SD}$ for triplicate transfections. $* \mathrm{P}<0.05 ; * * \mathrm{P}<0.01$ as compared with Mock by one-way analysis of variance.
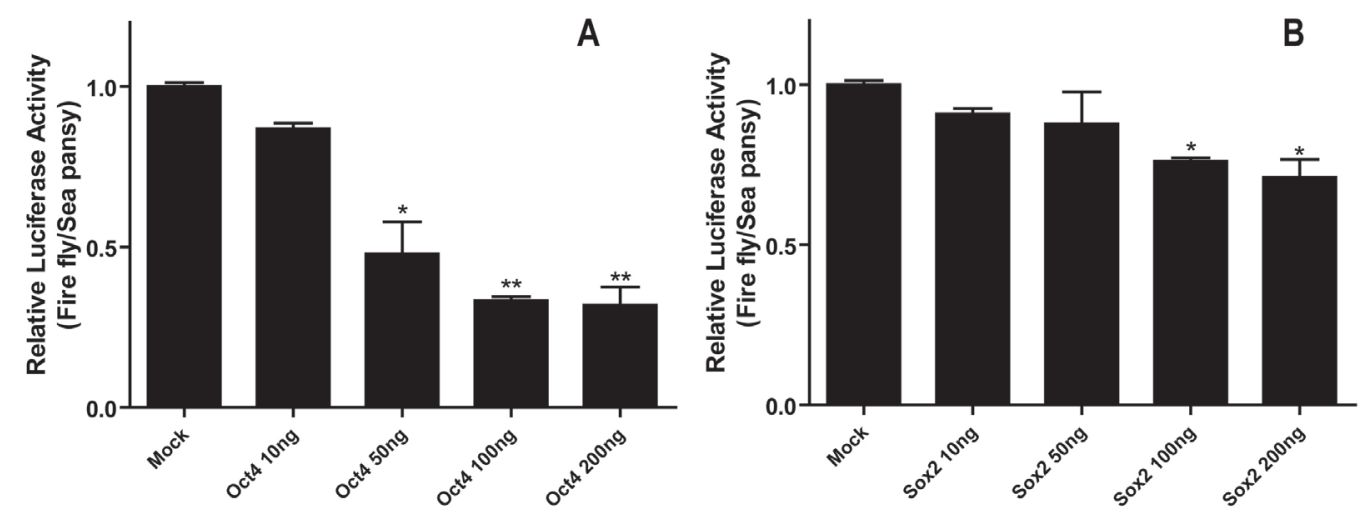

Figure 4. Dose dependence of Oct4 and Sox 2 on mouse Ezh2 promoter activity. First, 10-200 ng Oct4 (A) and Sox2 (B) were co-transfected with mouse Ezh2 promoter construct $(500 \mathrm{ng} /$ well) into NIH/3T3 cells. The protocol for transient transfections and luciferase assay is described in the figure legend of Figure 3 . ${ }^{*} \mathrm{P}<0.05, * * \mathrm{P}<0.01$ as compared with Mock by one-way analysis of variance. 


\section{DISCUSSION}

In this study, H3K27me3 modification was further confirmed in morulae and blastocysts of in vivo mouse embryos by immunofluorescence staining. We found that the intensity of $\mathrm{H} 3 \mathrm{~K} 27 \mathrm{me} 3$ in morulae was more extensive than that in blastocysts and that demethylation of H3K27me3 occurred during the morula stage. These results are consistent with those of a previous report (Zhang et al., 2009). Several studies have suggested that the promoters of inactive or weakly expressed genes are often enriched with H3K27me3, and these genes must undergo demethylation of H3K27me3 when expressed (Dahl et al., 2010). It is well known that many development-related genes are upregulated in blastocysts compared to morulae (Tanaka and Ko, 2004). Demethylation of H3K27me3 during the blastocyst stage was synchronized with the modification of H3K4me3 (Wu et al., 2012a), indicating that many developmentrelated genes were activated in this stage to maintain the progress of embryonic development and implantation during the latter stage.

The methytransferase activity of Ezh2 is closely associated with H3K27me3 in mammals (Zhu et al., 2011). mRNA expression of Ezh2 was observed in whole mouse embryos at day E9.5 and in the fetal liver, brain, and skeletal muscles at day E13 or day E17 (Laible et al., 1997). In the present study, the expression pattern of Ezh2 in mouse oocytes and in vivo preimplantation embryos were investigated by real-time PCR. Expression of Ezh2 was detected in oocytes, further indicating that Ezh2 is a maternal factor (Erhardt et al., 2003). During the entire preimplantation and embryo development periods, the highest level of Ezh2 peaked during the zygote stage and gradually decreased from the 2-cell stage onwards, while Ezh2 was expressed at the lowest level in blastocysts. The gradually decreasing Ezh2 mRNA expression may correspond to the gradually decreased enzymatic activity of Ezh2. These results are consistent with staining data obtained by immunofluorescence histochemistry. Therefore, Ezh2 may be involved in mouse embryonic development by mediating the modification of H3K27me3.

Oct4, Sox2, Nanog, Klf-4, and c-Myc correlated with the development potential of preimplantation embryos, the maintenance of ES cell pluripotency, and iPS cell totipotency (Li et al., 2005; Takahashi and Yamanaka, 2006; Suzuki et al., 2009). Oct4, Sox2, Nanog, and Klf-4 proteins were observed to be in the ICM cells of blastocysts; the fluorescence intensity increased with embryonic development, peaking during the blastocyst stage (Rodda et al., 2005; Zhang et al., 2009; Keramari et al., 2010). These gene expression profiles may have been caused by demethylation of H3K27 during the blastocyst stage. However, no c-Myc signal was detected in blastocysts (Suzuki et al., 2009). A previous study reported that the Ezh2 protein was observed in ICM cells according to immunofluorescence results (Zhang et al., 2009), indicating that Ezh2 and development-related genes are co-localized in mouse blastocysts. Using promoter analysis, we found that the promoter activity of Ezh2 was suppressed by Oct-4 and Sox 2 alone in NIH/3T3 cells in a dose-dependent manner. These results were consistent with the expression of Ezh2 determined by real-time PCR. In fact, this was not the first evidence that the transcription of epigenetic modifying enzyme genes is regulated by these master transcription factors. In mesenchymal stem cells, Oct4 and Nanog upregulated DNA methyltransferase 1 by directly binding to its promoter to maintain self-renewal and its undifferentiated state (Tsai et al., 2012). The H3K9me2 and H3K9me3 demethylase genes, Jmjd1a and $J m j d 2 c$, are positively regulated by Oct4 in ES cells (Loh et al., 2007). Ezh2 expression is controlled by Sox 4 during the epithelial-mesenchymal transition in normal and cancerous 
breast epithelial cells (Tiwari et al., 2013). In T47D cells, FOXP3 could downregulate Ezh2 protein level to promote mammosphere formation, cell growth, directional migration, and colony formation (Shen et al., 2013). These reports, together with our data, demonstrate that epigenetic modifying enzyme genes are direct transcriptional targets of key developmentrelated genes (Oct4 and Sox2). In contrast, the transcription activity of these developmentrelated genes also depended on the epigenetic modification catalyzed by epigenetic modifying enzymes.

Taken together, our study showed that Ezh2 mRNA expression was inversely correlated with Oct4 and Sox2, but Oct4 and Sox2 negatively affect Ezh2; additionally, Oct4 and Sox 2 were negative regulators of Ezh2 primarily on a post-translation level. These data illustrate the correlation between development-related genes and epigenetic modification in mouse embryonic development and cell reprograming.

\section{ACKNOWLEDGMENTS}

Research supported by grants from the National Natural Science Foundation of China (\#31372273, \#31071310, \#31201789), the Key Grant of the Natural Science Research Program of Anhui Higher Education Institutions of China (\#KJ2013A202), the Major Project of Discipline Construction in Anhui Province, the Natural Science Foundation of Anhui Province (\#1408085QC65), and the Research Program of Provincial Scientific Research Institutions (Grant \#2013PTFY04ZD).

\section{REFERENCES}

Boyer LA, Lee TI, Cole MF, Johnstone SE, et al. (2005). Core transcriptional regulatory circuitry in human embryonic stem cells. Cell 122: 947-956.

Dahl JA, Reiner AH, Klungland A, Wakayama T, et al. (2010). Histone H3 lysine 27 methylation asymmetry on developmentally-regulated promoters distinguish the first two lineages in mouse preimplantation embryos. PLoS One 5: e9150.

Erhardt S, Su IH, Schneider R, Barton S, et al. (2003). Consequences of the depletion of zygotic and embryonic enhancer of zeste 2 during preimplantation mouse development. Development 130: 4235-4248.

Fragola G, Germain PL, Laise P, Cuomo A, et al. (2013). Cell reprogramming requires silencing of a core subset of polycomb targets. PLoS Genet. 9: e1003292.

Keramari M, Razavi J, Ingman KA, Patsch C, et al. (2010). Sox2 is essential for formation of trophectoderm in the preimplantation embryo. PLoS One 5: e13952.

Laible G, Wolf A, Dorn R, Reuter G, et al. (1997). Mammalian homologues of the Polycomb-group gene Enhancer of zeste mediate gene silencing in Drosophila heterochromatin and at S. cerevisiae telomeres. EMBO J. 16: 3219-3232.

Li X, Kato Y and Tsunoda Y (2005). Comparative analysis of development-related gene expression in mouse preimplantation embryos with different developmental potential. Mol. Reprod. Dev. 72: 152-160.

Loh YH, Zhang W, Chen X, George J et al. (2007). Jmjd1a and Jmjd2c histone H3 Lys 9 demethylases regulate selfrenewal in embryonic stem cells. Genes Dev. 21: 2545-2557.

Park KE, Magnani L and Cabot RA (2009). Differential remodeling of mono- and trimethylated H3K27 during porcine embryo development. Mol. Reprod. Dev. 76: 1033-1042.

Qi W, Chan H, Teng L, Li L, et al. (2012). Selective inhibition of Ezh2 by a small molecule inhibitor blocks tumor cells proliferation. Proc. Natl. Acad. Sci. U. S. A. 109: 21360-21365.

Rodda DJ, Chew JL, Lim LH, Loh YH, et al. (2005). Transcriptional regulation of nanog by OCT4 and SOX2. J. Biol. Chem. 280: 24731-24737.

Sauvageau M and Sauvageau G (2010). Polycomb group proteins: multi-faceted regulators of somatic stem cells and cancer. Cell Stem Cell 7: 299-313.

Shen Z, Chen L, Yang X, Zhao Y, et al. (2013). Downregulation of Ezh2 methyltransferase by FOXP3: New insight of 
FOXP3 into chromatin remodeling? Biochim. Biophys. Acta 1833: 2190-2200.

Shin DM, Liu R, Wu W, Waigel SJ, et al. (2012). Global gene expression analysis of very small embryonic-like stem cells reveals that the Ezh2-dependent bivalent domain mechanism contributes to their pluripotent state. Stem Cells Dev. 21: $1639-1652$

Simon C, Chagraoui J, Krosl J, Gendron P, et al. (2012). A key role for Ezh2 and associated genes in mouse and human adult T-cell acute leukemia. Genes Dev. 26: 651-656.

Suzuki T, Abe K, Inoue A and Aoki F (2009). Expression of c-MYC in nuclear speckles during mouse oocyte growth and preimplantation development. J. Reprod. Dev. 55: 491-495.

Takahashi K and Yamanaka S (2006). Induction of pluripotent stem cells from mouse embryonic and adult fibroblast cultures by defined factors. Cell 126: 663-676.

Tanaka TS and Ko MS (2004). A global view of gene expression in the preimplantation mouse embryo: morulae versus blastocyst. Eur. J. Obstet. Gynecol. Reprod. Biol. 115 (Suppl 1): S85-S91

Tiwari N, Tiwari VK, Waldmeier L, Balwierz PJ, et al. (2013). Sox4 is a master regulator of epithelial-mesenchymal transition by controlling Ezh2 expression and epigenetic reprogramming. Cancer Cell 23: 768-783.

Tsai CC, Su PF, Huang YF, Yew TL, et al. (2012). Oct4 and Nanog directly regulate Dnmt1 to maintain self-renewal and undifferentiated state in mesenchymal stem cells. Mol. Cell 47: 169-182.

Villasante A, Piazzolla D, Li H, Gomez-Lopez G, et al. (2011). Epigenetic regulation of Nanog expression by Ezh2 in pluripotent stem cells. Cell Cycle 10: 1488-1498.

Wu FR, Ding B, Qi B, Shang MB, et al. (2012a). Sequence analysis, expression patterns and transcriptional regulation of mouse Ifrg 15 during preimplantation embryonic development. Gene 507: 119-124.

Wu FR, Liu Y, Shang MB, Yang XX, et al. (2012b). Differences in H3K4 trimethylation in in vivo and in vitro fertilization mouse preimplantation embryos. Genet. Mol. Res. 11: 1099-1108.

Zhang M, Wang F, Kou Z, Zhang Y, et al. (2009). Defective chromatin structure in somatic cell cloned mouse embryos. J. Biol. Chem. 284: 24981-24987.

Zhu NX, Liu SY, Su ZJ, Chen L, et al. (2011). The expression pattern of polycomb group protein Ezh2 during mouse embryogenesis. Anat. Rec. 294: 1150-1157. 